\title{
OPTIMIZATION ALGORITHM FOR RECONSTRUCTING INTERFACE CHANGES OF A CONDUCTIVITY INCLUSION FROM MODAL MEASUREMENTS
}

\author{
HABIB AMMARI, ELENA BERETTA, ELISA FRANCINI, HYEONBAE KANG, \\ AND MIKYOUNG LIM
}

\begin{abstract}
In this paper, we propose an original and promising optimization approach for reconstructing interface changes of a conductivity inclusion from measurements of eigenvalues and eigenfunctions associated with the transmission problem for the Laplacian. Based on a rigorous asymptotic analysis, we derive an asymptotic formula for the perturbations in the modal measurements that are due to small changes in the interface of the inclusion. Using fine gradient estimates, we carefully estimate the error term in this asymptotic formula. We then provide a key dual identity which naturally yields to the formulation of the proposed optimization problem. The viability of our reconstruction approach is documented by a variety of numerical results. The resolution limit of our algorithm is also highlighted.
\end{abstract}

\section{INTRODUCTION}

Let $\Omega$ be a smooth domain and $D$ an inclusion contained in $\Omega$ whose boundary is also assumed to be smooth. Shape deformation of $D$ causes a perturbation of modal parameters. The aim of this paper is to show how this information can be used to reconstruct the unknown deformation. For doing so, we rigorously derive an asymptotic formula for the perturbations in the eigenvalues of the transmission problem for the Laplacian that are due to small deformations of the interface of an inclusion. Based on this formula, we design an efficient reconstruction algorithm from modal measurements. Our algorithm consists of minimizing a functional whose minimizer yields certain geometric properties of the unknown inclusion. It naturally follows from a key identity that is in some sense dual to the asymptotic formula. Numerical experiments showing the viability of our algorithm are presented.

Our asymptotic formula for the perturbations in the eigenvalues due to changes of the shape of the inclusion $D$ that is inside a background domain $\Omega$ is in connection with the more classical ones established under variation of the background domain $\Omega$. There have been several interesting works on the eigenvalue perturbation problem under variation of the domain since the seminal formula of Hadamard [12. See for example the works by Garabedian and Schiffer [10, Kato [13], Sanchez Hubert

Received by the editor June 5, 2008 and, in revised form, March 25, 2009 and August 9, 2009. 2000 Mathematics Subject Classification. Primary 35R30, 35B34.

Key words and phrases. Shape reconstruction, vibration analysis, asymptotic expansion, reconstruction algorithm, optimization problem. 
and Sanchez Palencia [20, and Kozlov [14. Convergence results for the eigenvalues and eigenvectors under boundary variations have also been proved by abstract methods as, for example, those from [21, 22, 23].

Our results in this paper extend those established in the context of small volume inclusions as well as those for the conductivity interface problem. In fact, on one hand, in a series of recent papers [8, 6, 4, 5, we have derived high-order asymptotic expansions of the eigenvalue perturbations due to the presence of small inclusions and used them for locating the inclusions and identifying some of their geometric features. On the other hand, in [7, we have derived high-order terms in the asymptotic expansions of the boundary perturbations of steady-state voltage potentials resulting from small perturbations of the shape of a conductivity inclusion. Based on these derivations, we have designed an effective algorithm to determine some geometric features of the shape perturbation of the inclusion based on boundary measurements.

In this paper, the asymptotic formula for the perturbations in the modal measurements that are due to small changes in the interface of an inclusion is original. Fine gradient estimates are used for its derivation. Indeed, careful estimates of the error term in this formula are provided and a systematic way for deriving the dual identity that yields to the optimization problem is presented. The case of multiple eigenvalues is rigorously handled as well.

The paper is organized as follows. In the next section we derive an asymptotic formula for the eigenvalue perturbations due to shape deformation. We provide in Section 3 a functional whose minimizer yields the interface of the inclusion. For doing so, we provide a key dual identity which naturally yields to the formulation of the proposed optimization problem. In Section 4 , we consider the case of a multiple eigenvalue. In Section [5, we perform numerical experiments to test the viability of the algorithm. Many applications of our results in this paper are expected, especially in structural vibration testing [19].

\section{Asymptotic Formula}

Throughout this paper, let $\mathcal{C}^{k, \alpha}$ denote the Hölder space which consists of functions having derivatives up to order $k$ and such that the $k$ th derivative is Hölder continuous with exponent $\alpha$, where $0<\alpha \leq 1$. Let $\Omega \subset \mathbb{R}^{2}$ be a bounded domain with $\mathcal{C}^{2,1}$ boundary and let $D$ be an open subset of $\Omega$ such that $\operatorname{dist}(\partial \Omega, \partial D) \geq d_{0}>0$. The boundary $\partial D$ of $D$ is also assumed to be $\mathcal{C}^{2,1}$. Suppose that the conductivity (or the dielectric constant) of the background is $\gamma_{e}$ while that of the inclusion $D$ is $\gamma_{i}$. So the conductivity profile denoted by $\gamma_{D}$ is given by

$$
\gamma_{D}=\gamma_{e} \chi_{\Omega \backslash D}+\gamma_{i} \chi_{D},
$$

where $\chi_{D}$ is the characteristic function of $D$. Let $\left(u_{0}, \omega_{0}^{2}\right)$ be a solution of the following eigenvalue problem:

$$
\left\{\begin{aligned}
\nabla \cdot\left(\gamma_{D} \nabla u_{0}\right) & =-\omega_{0}^{2} u_{0} \quad \text { in } \quad \Omega \\
\gamma_{D} \frac{\partial u_{0}}{\partial \nu} & =0 \quad \text { on } \quad \partial \Omega \\
\int_{\Omega} u_{0}^{2} & =1
\end{aligned}\right.
$$


Let

$$
u_{0}^{e}=\left.u_{0}\right|_{\Omega \backslash D} \quad \text { and } \quad u_{0}^{i}=\left.u_{0}\right|_{\bar{D}} .
$$

Then along the interface $\partial D$, the following transmission conditions hold:

$$
\left\{\begin{aligned}
u_{0}^{i} & =u_{0}^{e} \\
\gamma_{i} \frac{\partial u_{0}^{i}}{\partial \nu} & =\gamma_{e} \frac{\partial u_{0}^{e}}{\partial \nu}
\end{aligned} \text { on } \partial D,\right.
$$

where $\frac{\partial}{\partial \nu}$ denotes the normal derivative with respect to the outward unit normal to $\partial D$. The first condition in (4) represents the continuity of the potential while the second one represents the flux. We emphasize that since $u_{0}^{i}$ and $u_{0}^{e}$ are $\mathcal{C}^{1, \alpha}$ for some $0<\alpha<1$, as will be proven in the next section, these conditions hold in the pointwise sense. From the first condition, we also have

$$
\frac{\partial u_{0}^{i}}{\partial \tau}=\frac{\partial u_{0}^{e}}{\partial \tau}\left(=\frac{\partial u_{0}}{\partial \tau}\right)
$$

where $\frac{\partial}{\partial \tau}$ denotes the tangential derivative along $\partial D$.

In this section as well as in the next one, we will assume that $\omega_{0}^{2}$ is a simple eigenvalue. This will make our arguments more readable. In Section 4 we will state our result in the case of multiple eigenvalues.

Now let us consider $D_{\varepsilon}$ an $\varepsilon$-perturbation of the domain $D$ with

$$
\partial D_{\varepsilon}=\{\tilde{x}: \tilde{x}=x+\varepsilon h(x) \nu(x), x \in \partial D\},
$$

where $\nu(x)$ is the unit outer normal vector to $\partial D$ at $x, h \in \mathcal{C}^{1,1}(\partial D)$ with $\|h\|_{\mathcal{C}^{1,1}} \leq$ $H$ for some positive constant $H$, and $\varepsilon$ is a positive small parameter.

Let $\gamma_{D_{\varepsilon}}=\gamma_{e} \chi_{\Omega \backslash D_{\varepsilon}}+\gamma_{i} \chi_{D_{\varepsilon}}$ and consider the following eigenvalue problem on the perturbed domain:

$$
\left\{\begin{aligned}
\nabla \cdot\left(\gamma_{D_{\varepsilon}} \nabla u_{\varepsilon}\right) & =-\omega_{\varepsilon}^{2} u_{\varepsilon} \quad \text { in } \quad \Omega, \\
\gamma_{D_{\varepsilon}} \frac{\partial u_{\varepsilon}}{\partial \nu} & =0 \quad \text { on } \quad \partial \Omega, \\
\int_{\Omega} u_{\varepsilon}^{2} & =1 .
\end{aligned}\right.
$$

Our main result in this section is the following theorem.

Theorem 2.1. Let $\omega_{0}^{2}$ be a simple eigenvalue of (2). Then there exists a simple eigenvalue of (6), denoted by $\omega_{\varepsilon}^{2}$, such that $\omega_{\varepsilon}^{2} \rightarrow \omega_{0}^{2}$ as $\varepsilon \rightarrow 0$, and the following asymptotic expansion holds:

$$
\omega_{\varepsilon}^{2}-\omega_{0}^{2}=-\varepsilon\left(\gamma_{i}-\gamma_{e}\right) \int_{\partial D} h(x)\left(\left(\frac{\partial u_{0}^{e}}{\partial \tau}(x)\right)^{2}+\frac{\gamma_{e}}{\gamma_{i}}\left(\frac{\partial u_{0}^{e}}{\partial \nu}(x)\right)^{2}\right) d \sigma_{x}+O\left(\varepsilon^{1+\beta}\right)
$$

for some $\beta>0$, where $\left(u_{0}, \omega_{0}^{2}\right)$ is the solution to (2).

It is worth noting that if $h$ has a constant sign on $\partial D$, then there exists $\varepsilon_{0}>0$ such that for $\varepsilon<\varepsilon_{0}, \omega_{\varepsilon}^{2}-\omega_{0}^{2}$ has the same sign as $\left(\gamma_{e}-\gamma_{i}\right) h$.

We will prove Theorem 2.1] using Osborn's result in [17] concerning estimates for the eigenvalues of a sequence of self-adjoint compact operators. More precisely, let $X$ be a real Hilbert space and let $T: X \rightarrow X$ and $T_{\varepsilon}: X \rightarrow X$ be compact, self-adjoint linear operators such that $\left\{T_{\varepsilon}\right\}_{\varepsilon>0}$ are collectively compact and $T_{\varepsilon} \rightarrow T$ 
pointwise as $\varepsilon \rightarrow 0$. Let $\mu_{0}$ be a nonzero eigenvalue of $T$ with multiplicity $m$. Then, for $\varepsilon$ small, $T_{\varepsilon}$ has a set of $m$ eigenvalues (counted according to their multiplicity) such that $\mu_{\varepsilon}^{j} \rightarrow \mu_{0}$ for each $j=1, \ldots, m$, as $\varepsilon \rightarrow 0$. Let $\bar{\mu}_{\varepsilon}=\frac{1}{m} \sum_{j=1}^{m} \mu_{\varepsilon}^{j}$. If $\left\{u_{0,1}, u_{0,2}, \ldots, u_{0, m}\right\}$ is an orthonormal basis for $\operatorname{Ker}\left(T-\mu_{0} I\right)$, then there exists a constant $C$ (independent of $\varepsilon$ ) such that

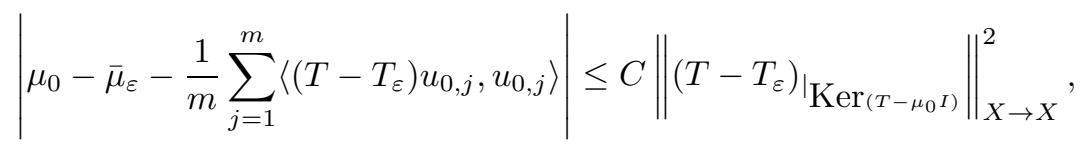

where the right-hand side of (8) denotes the operator norm of $T-T_{\varepsilon}$ on the subspace $\operatorname{Ker}\left(T-\mu_{0} I\right) \subset X$. Moreover, for each $j=1, \ldots, m$, there is an eigenfunction $u_{\varepsilon, j}$ corresponding to $\mu_{\varepsilon}^{j}$, such that $\left\|u_{\varepsilon, j}\right\|_{X}=1$, and

$$
\left\|u_{\varepsilon, j}-u_{0, j}\right\|_{X} \leq C\left\|\left(T-T_{\varepsilon}\right)_{\mid} \operatorname{Ker}_{\left(T-\mu_{0} I\right)}\right\|_{X \rightarrow X} .
$$

If $\mu_{0}$ is a simple eigenvalue, for $\varepsilon$ small, there is a simple eigenvalue $\mu_{\varepsilon}$ for $T_{\varepsilon}$ such that

$$
\left|\mu_{0}-\mu_{\varepsilon}-\left\langle\left(T-T_{\varepsilon}\right) u_{0}, u_{0}\right\rangle\right| \leq C\left\|\left(T-T_{\varepsilon}\right)\left(u_{0}\right)\right\|_{X}^{2} .
$$

Furthermore, let $u_{\varepsilon}$ be the eigenfunction corresponding to $\mu_{\varepsilon}$ such that $\left\|u_{\varepsilon}\right\|_{X}=1$, then

$$
\left\|u_{\varepsilon}-u_{0}\right\|_{X} \leq C\left\|\left(T-T_{\varepsilon}\right)\left(u_{0}\right)\right\|_{X} .
$$

Let us consider $X=\left\{f \in L^{2}(\Omega): \int_{\Omega} f=0\right\}$ with the usual inner product of $L^{2}(\Omega)$ and $T: X \rightarrow X$ the linear operator given by $T f=v_{0}$ where $v_{0}$ is the solution to

$$
\left\{\begin{aligned}
\nabla \cdot\left(\gamma_{D} \nabla v_{0}\right) & =f \quad \text { in } \quad \Omega, \\
\gamma_{D} \frac{\partial v_{0}}{\partial \nu} & =0 \quad \text { on } \quad \partial \Omega, \\
\int_{\Omega} v_{0} & =0 .
\end{aligned}\right.
$$

We define $T_{\varepsilon}: X \rightarrow X$ similarly, i.e., by $T_{\varepsilon} f=v_{\varepsilon}$, where $v_{\varepsilon}$ is the solution to

$$
\left\{\begin{aligned}
\nabla \cdot\left(\gamma_{D_{\varepsilon}} \nabla v_{\varepsilon}\right) & =f \quad \text { in } \quad \Omega, \\
\gamma_{D_{\varepsilon}} \frac{\partial v_{\varepsilon}}{\partial \nu} & =0 \quad \text { on } \quad \partial \Omega, \\
\int_{\Omega} v_{\varepsilon} & =0 .
\end{aligned}\right.
$$

Then $T$ and $T_{\varepsilon}$ are compact self-adjoint operators. We now prove that $\left\{T_{\varepsilon}\right\}_{\varepsilon \geq 0}$, $\left(T_{0}=T\right)$ are collectively compact and that $T_{\varepsilon} \rightarrow T$ pointwise as $\varepsilon \rightarrow 0$.

(i) $\left\{T_{\varepsilon}\right\}_{\varepsilon \geq 0}$ are collectively compact, i.e. $\left\{T_{\varepsilon} f:\|f\|_{X} \leq 1, \varepsilon \geq 0\right\}$ is sequentially compact: If $v \in\left\{T_{\varepsilon} f:\|f\|_{X} \leq 1, \varepsilon \geq 0\right\}$, then from energy estimates and the Poincaré inequality we have that $\|v\|_{H^{1}(\Omega)} \leq C$ where $C$ is independent of $\varepsilon$. Since $H^{1}(\Omega)$ is compactly embedded in $L^{2}(\Omega)$, this guarantees that $\left\{T_{\varepsilon} f:\|f\|_{X} \leq 1, \varepsilon \geq 0\right\}$ is sequentially compact in $L^{2}(\Omega)$. 
(ii) $T_{\varepsilon} \rightarrow T$ pointwise as $\varepsilon \rightarrow 0$ in $X$ : For $f \in X$, let $v_{\varepsilon}=T_{\varepsilon} f$ and $v_{0}=T f$. Then, for any $w \in H^{1}(\Omega)$,

$$
\int_{\Omega} \gamma_{D_{\varepsilon}} \nabla v_{\varepsilon} \cdot \nabla w=-\int_{\Omega} f w
$$

and

$$
\int_{\Omega} \gamma_{D} \nabla v_{0} \cdot \nabla w=-\int_{\Omega} f w
$$

Hence, choosing $w=v_{\varepsilon}-v_{0}$ and subtracting these two equations we get

$$
\int_{\Omega}\left(\gamma_{D_{\varepsilon}} \nabla v_{\varepsilon}-\gamma_{D} \nabla v_{0}\right) \cdot \nabla\left(v_{\varepsilon}-v_{0}\right)=0
$$

which gives

$$
\int_{\Omega} \gamma_{D_{\varepsilon}} \nabla\left(v_{\varepsilon}-v_{0}\right) \cdot \nabla\left(v_{\varepsilon}-v_{0}\right)=-\int_{\Omega}\left(\gamma_{D_{\varepsilon}}-\gamma_{D}\right) \nabla v_{0} \cdot \nabla\left(v_{\varepsilon}-v_{0}\right) .
$$

Hence

$$
\left\|\nabla\left(v_{\varepsilon}-v_{0}\right)\right\|_{L^{2}(\Omega)}^{2} \leq C\left\|\nabla v_{0}\right\|_{L^{2}\left(D_{\varepsilon} \triangle D\right)}\left\|\nabla\left(v_{\varepsilon}-v_{0}\right)\right\|_{L^{2}(\Omega)},
$$

where $\triangle$ denotes the symmetric difference, which implies

$$
\left\|\nabla\left(v_{\varepsilon}-v_{0}\right)\right\|_{L^{2}(\Omega)} \leq C\left\|\nabla v_{0}\right\|_{L^{2}\left(D_{\varepsilon} \triangle D\right)} .
$$

It then follows by the Poincaré inequality that

$$
\left\|v_{\varepsilon}-v_{0}\right\|_{H^{1}(\Omega)} \leq C\left\|\nabla v_{0}\right\|_{L^{2}\left(D_{\varepsilon} \triangle D\right)} .
$$

Finally, using the last inequality and the fact that $\left|D_{\varepsilon} \triangle D\right| \rightarrow 0$ as $\varepsilon \rightarrow 0$ and that $\nabla v_{0} \in L^{2}(\Omega)$ we obtain that $T_{\varepsilon} \rightarrow T$ pointwise as $\varepsilon \rightarrow 0$ in $L^{2}(\Omega)$.

We can now apply Osborn's result to conclude that, for small $\varepsilon$, there is an eigenvalue $\mu_{\varepsilon}$ of $T_{\varepsilon}$ such that $\mu_{\varepsilon} \rightarrow \mu_{0}$ and

$$
\left|\mu_{0}-\mu_{\varepsilon}-\left\langle\left(T_{\varepsilon}-T\right) u_{0}, u_{0}\right\rangle\right| \leq C\left\|\left(T_{\varepsilon}-T\right) u_{0}\right\|_{L^{2}(\Omega)}^{2},
$$

where $u_{0}$ is such that $T u_{0}=\mu_{0} u_{0}$ and $\int_{\Omega} u_{0}^{2}=1$. (Note that the compatibility condition $\int_{\Omega} u_{0}=0$ is also satisfied). Moreover,

$$
\left\|u_{\varepsilon}-u_{0}\right\|_{L^{2}(\Omega)} \leq C\left\|\left(T_{\varepsilon}-T\right) u_{0}\right\|_{L^{2}(\Omega)}
$$

where $u_{\varepsilon}$ is the eigenfunction corresponding to $\mu_{\varepsilon}$ such that $\int_{\Omega} u_{\varepsilon}^{2}=1$. The eigenfunctions $u_{0}$ and $u_{\varepsilon}$ solve, respectively, the problems

$$
\left\{\begin{aligned}
\nabla \cdot\left(\gamma_{D} \nabla u_{0}\right) & =\frac{u_{0}}{\mu_{0}} \quad \text { in } \quad \Omega \\
\gamma_{D} \frac{\partial u_{0}}{\partial \nu} & =0 \quad \text { on } \quad \partial \Omega \\
\int_{\Omega} u_{0}^{2} & =1
\end{aligned}\right.
$$

and

$$
\left\{\begin{aligned}
\nabla \cdot\left(\gamma_{D_{\varepsilon}} \nabla u_{\varepsilon}\right) & =\frac{u_{\varepsilon}}{\mu_{\varepsilon}} \quad \text { in } \quad \Omega, \\
\gamma_{D_{\varepsilon}} \frac{\partial u_{\varepsilon}}{\partial \nu} & =0 \quad \text { on } \quad \partial \Omega \\
\int_{\Omega} u_{\varepsilon}^{2} & =1 .
\end{aligned}\right.
$$


Let us now consider some regularity facts on the functions $u_{\varepsilon}$ and $u_{0}$. From [16], it follows that, for some $\alpha \in(0,1), u_{0} \in \mathcal{C}^{1, \alpha}(\bar{D}) \cap \mathcal{C}^{1, \alpha}(\Omega \backslash \bar{D})$ and

$$
\left\|u_{0}\right\|_{\mathcal{C}^{1, \alpha}(\bar{D})} \leq C\left(\left\|u_{0}\right\|_{L^{2}(\Omega)}+\left\|u_{0}\right\|_{L^{\infty}(\Omega)}\right)
$$

where $C=C\left(\gamma_{e}, \gamma_{i}, \Omega, D, \mu_{0}, \alpha\right)$ and analogously, since $\operatorname{dist}(\partial \Omega, \partial D) \geq d_{0}>0$,

$$
\left\|u_{0}\right\|_{\mathcal{C}^{1, \alpha}\left(\Omega_{d_{0} / 2} \backslash D\right)} \leq C\left(\left\|u_{0}\right\|_{L^{2}(\Omega)}+\left\|u_{0}\right\|_{L^{\infty}(\Omega)}\right),
$$

where $C=C\left(\gamma_{e}, \gamma_{i}, \Omega, D, \mu_{0}, d_{0}, \alpha\right)$ and $\Omega_{d_{0} / 2}=\left\{x \in \Omega: \operatorname{dist}(x, \partial \Omega)>d_{0} / 2\right\}$. Note that the right-hand sides in (18) and (19) are bounded by $C\|u\|_{L^{\infty}(\Omega)}$. Recalling that $u_{0}$ is the solution of a homogeneous Neumann problem we have, by global estimates for weak solutions of elliptic equations in divergence form with bounded coefficients (De Giorgi-Nash method, cf. [11, Theorem 8.24]), that

$$
\left\|u_{0}\right\|_{L^{\infty}(\Omega)} \leq C\left\|u_{0}\right\|_{L^{2}(\Omega)} .
$$

It then follows from (18) and (19) that

$$
\left\|u_{0}\right\|_{\mathcal{C}^{1, \alpha}(\bar{D})} \leq C\left\|u_{0}\right\|_{L^{2}(\Omega)},
$$

where $C=C\left(\gamma_{e}, \gamma_{i}, \Omega, D, \mu_{0}\right)$ and

$$
\left\|u_{0}\right\|_{\mathcal{C}^{1, \alpha}\left(\Omega_{d_{0} / 2} \backslash D\right)} \leq C\left\|u_{0}\right\|_{L^{2}(\Omega)},
$$

where $C=C\left(\gamma_{e}, \gamma_{i}, \Omega, D, \mu_{0}, d_{0}\right)$. Since $\left\|u_{0}\right\|_{L^{2}(\Omega)}=1$, we have that

$$
\left\|u_{0}\right\|_{\mathcal{C}^{1, \alpha}(\bar{D})} \leq C
$$

and

$$
\left\|u_{0}\right\|_{\mathcal{C}^{1, \alpha}\left(\Omega_{d_{0} / 2} \backslash D\right)} \leq C .
$$

Similarly, we get for $u_{\varepsilon}$,

$$
\left\|u_{\varepsilon}\right\|_{\mathcal{C}^{1, \alpha}\left(\bar{D}_{\varepsilon}\right)} \leq C
$$

where $C=C\left(\gamma_{e}, \gamma_{i}, \Omega, D, \mu_{0}, H, \alpha\right)$ and

$$
\left\|u_{\varepsilon}\right\|_{\mathcal{C}^{1, \alpha}\left(\Omega_{d_{0} / 2} \backslash D_{\varepsilon}\right)} \leq C
$$

where here $C=C\left(\gamma_{e}, \gamma_{i}, \Omega, D, \mu_{0}, d_{0}, H, \alpha\right)$. It is worth emphasizing that the constant $C$ in all four of the above estimates is independent of $\varepsilon$.

Let us now evaluate the right-hand side of inequality (15). We know that $T u_{0}=$ $\mu_{0} u_{0}$ and $T_{\varepsilon} u_{0}=\tilde{v}_{\varepsilon}$, where $\tilde{v}_{\varepsilon}$ is the solution to

$$
\left\{\begin{aligned}
\nabla \cdot\left(\gamma_{D_{\varepsilon}} \nabla \tilde{v}_{\varepsilon}\right) & =u_{0} \quad \text { in } \quad \Omega \\
\gamma_{D_{\varepsilon}} \frac{\partial \tilde{v}_{\varepsilon}}{\partial \nu} & =0 \quad \text { on } \quad \partial \Omega \\
\int_{\Omega} \tilde{v}_{\varepsilon} & =0
\end{aligned}\right.
$$

Since $u_{0}$ satisfies (16), we may use exactly the same argument as the one for deriving (13) to show that

$$
\left\|\tilde{v}_{\varepsilon}-\mu_{0} u_{0}\right\|_{H^{1}(\Omega)} \leq C\left\|\nabla u_{0}\right\|_{L^{2}\left(D_{\varepsilon} \triangle D\right)}
$$

It then follows from (21) and (22) that

$$
\left\|\tilde{v}_{\varepsilon}-\mu_{0} u_{0}\right\|_{H^{1}(\Omega)} \leq C\left|D_{\varepsilon} \triangle D\right|^{1 / 2} .
$$


Observe that $\tilde{v}_{\varepsilon}-\mu_{0} u_{0}$ is a solution to

$$
\left\{\begin{aligned}
\nabla \cdot\left(\gamma_{D_{\varepsilon}} \nabla\left(\tilde{v}_{\varepsilon}-\mu_{0} u_{0}\right)\right) & =\mu_{0} \nabla \cdot\left(\left(\gamma_{D}-\gamma_{D_{\varepsilon}}\right) \nabla u_{0}\right) \quad \text { in } \quad \Omega, \\
\gamma_{D_{\varepsilon}} \frac{\partial\left(\tilde{v}_{\varepsilon}-\mu_{0} u_{0}\right)}{\partial \nu} & =0 \quad \text { on } \quad \partial \Omega \\
\int_{\Omega}\left(\tilde{v}_{\varepsilon}-\mu_{0} u_{0}\right) & =0 .
\end{aligned}\right.
$$

Applying Lemma A.1 in [2] for $V=\left\{v \in H^{1}(\Omega): \int_{\Omega} v=0\right\}, \phi=\tilde{v}_{\varepsilon}-\mu_{0} u_{0}$ and $F=\mu_{0}\left(\gamma_{D}-\gamma_{D_{\varepsilon}}\right) \nabla u_{0}$ we then get

$$
\left\|\tilde{v}_{\varepsilon}-\mu_{0} u_{0}\right\|_{L^{2}(\Omega)} \leq C\left|D_{\varepsilon} \triangle D\right|^{1 / 2+\eta},
$$

for some $\eta>0$ where $C=C\left(\gamma_{e}, \gamma_{i}, \mu_{0}, \Omega, H, \eta\right)$ but is otherwise independent of $\varepsilon$. Hence we have

$$
\left\|\left(T_{\varepsilon}-T\right) u_{0}\right\|_{L^{2}(\Omega)} \leq C \varepsilon^{1 / 2+\eta}
$$

and from (15)

$$
\left\|u_{\varepsilon}-u_{0}\right\|_{L^{2}(\Omega)} \leq C \varepsilon^{1 / 2+\eta} .
$$

Furthermore, observing that $\left\|\tilde{v}_{\varepsilon}\right\|_{H^{1}(\Omega)} \leq\left\|u_{0}\right\|_{L^{2}(\Omega)}$ and using the gradient estimates of [16], we obtain

$$
\left\|\tilde{v}_{\varepsilon}\right\|_{\mathcal{C}^{1, \alpha}\left(\bar{D}_{\varepsilon}\right)} \leq C
$$

and analogously

$$
\left\|\tilde{v}_{\varepsilon}\right\|_{\mathcal{C}^{1, \alpha}\left(\Omega_{d_{0} / 2} \backslash D_{\varepsilon}\right)} \leq C,
$$

where $C=C\left(\gamma_{e}, \gamma_{i}, \Omega, D, \mu_{0}, d_{0}, H, \alpha\right)$.

As in (3), let us put

$$
\tilde{v}_{\varepsilon}^{e}:=\left.\tilde{v}_{\varepsilon}\right|_{\Omega \backslash D_{\varepsilon}} \quad \text { and } \quad \tilde{v}_{\varepsilon}^{i}:=\left.\tilde{v}_{\varepsilon}\right|_{D_{\varepsilon}} .
$$

The following lemma holds.

Lemma 2.2. Let $\alpha>0$ be the same Hölder exponent as in (29). There exists a constant $C$ independent of $\varepsilon$ such that

$$
\left\|\nabla\left(\tilde{v}_{\varepsilon}^{e}-\mu_{0} u_{0}^{e}\right)\right\|_{L^{\infty}\left(\partial D_{\varepsilon} \backslash D\right)}+\left\|\nabla\left(\tilde{v}_{\varepsilon}^{i}-\mu_{0} u_{0}^{i}\right)\right\|_{L^{\infty}\left(\partial D_{\varepsilon} \cap \bar{D}\right)} \leq C \varepsilon^{\frac{\alpha}{2 \alpha+2}},
$$

where $C=C\left(\gamma_{e}, \gamma_{i}, \Omega, D, \mu_{0}, d_{0}, H, \alpha\right)$.

Proof. Let $2 \varepsilon<d<d_{0} / 2$ and let $\Omega_{d}^{\varepsilon}=\left\{x \in \Omega \backslash\left(D \cup D_{\varepsilon}\right): \operatorname{dist}\left(x, \partial\left(\Omega \backslash D \cup D_{\varepsilon}\right)\right)>\right.$ $d\}$. Since $\nabla\left(\tilde{v}_{\varepsilon}-\mu_{0} u_{0}\right)$ is harmonic in $\Omega \backslash D \cup D_{\varepsilon}$ we may apply the mean value theorem to points $y \in \Omega_{d}^{\varepsilon}$ :

$$
\nabla\left(\tilde{v}_{\varepsilon}^{e}-\mu_{0} u_{0}^{e}\right)(y)=\frac{1}{\left|B_{d / 2}\right|} \int_{B_{d / 2}(y)} \nabla\left(\tilde{v}_{\varepsilon}-\mu_{0} u_{0}\right) d x
$$

to get

$$
\begin{aligned}
\left\|\nabla\left(\tilde{v}_{\varepsilon}^{e}-\mu_{0} u_{0}^{e}\right)\right\|_{L^{\infty}\left(\Omega_{d}^{\varepsilon}\right)} & \leq C d^{-1}\left\|\nabla\left(\tilde{v}_{\varepsilon}-\mu_{0} u_{0}\right)\right\|_{L^{2}(\Omega)} \\
& \leq C d^{-1} \varepsilon^{1 / 2}
\end{aligned}
$$

Now, let $y \in \partial D_{\varepsilon} \backslash D$ and let $y_{d}$ denote the closest point to $y$ in the set $\Omega_{d}^{\varepsilon}$. By (29) we obtain

$$
\left|\nabla \tilde{v}_{\varepsilon}^{e}(y)-\nabla \tilde{v}_{\varepsilon}^{e}\left(y_{d}\right)\right| \leq C d^{\alpha}
$$


Combining (31) and (32) gives

$$
\begin{aligned}
\left|\nabla\left(\tilde{v}_{\varepsilon}^{e}-\mu_{0} u_{0}^{e}\right)(y)\right| \leq & \left|\nabla \tilde{v}_{\varepsilon}^{e}(y)-\nabla \tilde{v}_{\varepsilon}^{e}\left(y_{d}\right)\right|+\left|\nabla \tilde{v}_{\varepsilon}^{e}\left(y_{d}\right)-\nabla\left(\mu_{0} u_{0}^{e}\right)\left(y_{d}\right)\right| \\
& +\left|\nabla \mu_{0} u_{0}^{e}\left(y_{d}\right)-\nabla \mu_{0} u_{0}^{e}(y)\right| \\
\leq & C\left(d^{\alpha}+d^{-1} \varepsilon^{1 / 2}\right) .
\end{aligned}
$$

Here we also used the gradient estimates for $u_{0}$. By choosing $d=\varepsilon^{\frac{1}{2(\alpha+1)}}$ we get

$$
\left\|\nabla\left(\tilde{v}_{\varepsilon}^{e}-\mu_{0} u_{0}^{e}\right)\right\|_{L^{\infty}\left(\partial D_{\varepsilon} \backslash D\right)} \leq C \varepsilon^{\frac{\alpha}{2 \alpha+2}} .
$$

In a similar way one can prove that

$$
\left\|\nabla\left(\tilde{v}_{\varepsilon}^{i}-\mu_{0} u_{0}^{i}\right)\right\|_{L^{\infty}\left(\partial D_{\varepsilon} \cap D\right)} \leq C \varepsilon^{\frac{\alpha}{2 \alpha+2}}
$$

to complete the proof of the lemma.

We are now ready to compute the term $\left\langle\left(T-T_{\varepsilon}\right) u_{0}, u_{0}\right\rangle$ in (14). We proceed with

$$
\begin{aligned}
\left\langle\left(T-T_{\varepsilon}\right) u_{0}, u_{0}\right\rangle & =\left\langle\mu_{0} u_{0}-\tilde{v}_{\varepsilon}, u_{0}\right\rangle \\
& =\mu_{0} \int_{\Omega} u_{0}^{2}-\int_{\Omega} u_{0} \tilde{v}_{\varepsilon} \\
& =-\mu_{0} \int_{\Omega}\left(\gamma_{D_{\varepsilon}}-\gamma_{D}\right) \nabla \tilde{v}_{\varepsilon} \cdot \nabla u_{0} \\
& =-\mu_{0} \int_{D_{\varepsilon} \backslash D}\left(\gamma_{i}-\gamma_{e}\right) \nabla \tilde{v}_{\varepsilon}^{i} \cdot \nabla u_{0}^{e}+\mu_{0} \int_{D \backslash D_{\varepsilon}}\left(\gamma_{i}-\gamma_{e}\right) \nabla \tilde{v}_{\varepsilon}^{e} \cdot \nabla u_{0}^{i} .
\end{aligned}
$$

Let $x_{t}:=x+\operatorname{th}(x) \nu(x)$ for $x \in \partial D$ and $t \in[0, \varepsilon]$. Then the Jacobian determinant of the change of variables $(x, t) \in \partial D \times[0, \varepsilon] \mapsto x_{t} \in D_{\varepsilon} \triangle D$ is $|h(x)|+O(\varepsilon)$ for $\varepsilon$ small enough, and hence we get

$$
\begin{aligned}
& -\mu_{0} \int_{D_{\varepsilon} \backslash D}\left(\gamma_{i}-\gamma_{e}\right) \nabla \tilde{v}_{\varepsilon}^{i} \cdot \nabla u_{0}^{e} d x \\
& =-\mu_{0} \int_{0}^{\varepsilon} \int_{\partial D \cap\{h>0\}} h(x)\left(\gamma_{i}-\gamma_{e}\right) \nabla \tilde{v}_{\varepsilon}^{i}\left(x_{t}\right) \cdot \nabla u_{0}^{e}\left(x_{t}\right) d \sigma_{x} d t+O\left(\varepsilon^{2}\right)
\end{aligned}
$$

and

$$
\begin{aligned}
& \mu_{0} \int_{D \backslash D_{\varepsilon}}\left(\gamma_{i}-\gamma_{e}\right) \nabla \tilde{v}_{\varepsilon}^{e} \cdot \nabla u_{0}^{i} d x \\
& =-\mu_{0} \int_{0}^{\varepsilon} \int_{\partial D \cap\{h<0\}} h(x)\left(\gamma_{i}-\gamma_{e}\right) \nabla \tilde{v}_{\varepsilon}^{e}\left(x_{t}\right) \cdot \nabla u_{0}^{i}\left(x_{t}\right) d \sigma_{x} d t+O\left(\varepsilon^{2}\right) .
\end{aligned}
$$

Using the gradient estimates (21), (22), (28), and (29), we have for $t \in[0, \varepsilon]$,

$$
\begin{aligned}
\nabla \tilde{v}_{\varepsilon}^{i}\left(x_{t}\right) \cdot \nabla u_{0}^{e}\left(x_{t}\right) & =\nabla \tilde{v}_{\varepsilon}^{i}\left(x_{\varepsilon}\right) \cdot \nabla u_{0}^{e}\left(x_{\varepsilon}\right)+O\left(\varepsilon^{\alpha}\right) \\
& =\frac{\partial \tilde{v}_{\varepsilon}^{i}}{\partial \tau}\left(x_{\varepsilon}\right) \frac{\partial u_{0}^{e}}{\partial \tau}\left(x_{\varepsilon}\right)+\frac{\partial \tilde{v}_{\varepsilon}^{i}}{\partial \nu}\left(x_{\varepsilon}\right) \frac{\partial u_{0}^{e}}{\partial \nu}\left(x_{\varepsilon}\right)+O\left(\varepsilon^{\alpha}\right) \\
& =\frac{\partial \tilde{v}_{\varepsilon}^{e}}{\partial \tau}\left(x_{\varepsilon}\right) \frac{\partial u_{0}^{e}}{\partial \tau}\left(x_{\varepsilon}\right)+\frac{\gamma_{e}}{\gamma_{i}} \frac{\partial \tilde{v}_{\varepsilon}^{e}}{\partial \nu}\left(x_{\varepsilon}\right) \frac{\partial u_{0}^{e}}{\partial \nu}\left(x_{\varepsilon}\right)+O\left(\varepsilon^{\alpha}\right) .
\end{aligned}
$$


Here we used the transmission conditions (44) and (5). We then use (22) and (30) to get

$$
\begin{aligned}
\nabla \tilde{v}_{\varepsilon}^{i}\left(x_{t}\right) \cdot \nabla u_{0}^{e}\left(x_{t}\right) & =\mu_{0} \frac{\partial u_{0}^{e}}{\partial \tau}\left(x_{\varepsilon}\right) \frac{\partial u_{0}^{e}}{\partial \tau}\left(x_{\varepsilon}\right)+\mu_{0} \frac{\gamma_{e}}{\gamma_{i}} \frac{\partial u_{0}^{e}}{\partial \nu}\left(x_{\varepsilon}\right) \frac{\partial u_{0}^{e}}{\partial \nu}\left(x_{\varepsilon}\right)+O\left(\varepsilon^{\frac{\alpha}{2 \alpha+2}}\right) \\
& =\mu_{0} \frac{\partial u_{0}^{e}}{\partial \tau}(x) \frac{\partial u_{0}^{e}}{\partial \tau}(x)+\mu_{0} \frac{\gamma_{e}}{\gamma_{i}} \frac{\partial u_{0}^{e}}{\partial \nu}(x) \frac{\partial u_{0}^{e}}{\partial \nu}(x)+O\left(\varepsilon^{\frac{\alpha}{2 \alpha+2}}\right) .
\end{aligned}
$$

It then follows from (33) that

$$
\begin{aligned}
& -\mu_{0} \int_{D_{\varepsilon} \backslash D}\left(\gamma_{i}-\gamma_{e}\right) \nabla \tilde{v}_{\varepsilon}^{i} \cdot \nabla u_{0}^{e} d x \\
& =-\mu_{0}^{2} \varepsilon\left(\gamma_{i}-\gamma_{e}\right) \int_{\partial D \cap\{h>0\}} h\left[\left(\frac{\partial u_{0}^{e}}{\partial \tau}\right)^{2}+\frac{\gamma_{e}}{\gamma_{i}}\left(\frac{\partial u_{0}^{e}}{\partial \nu}\right)^{2}\right] d \sigma+O\left(\varepsilon^{1+\beta}\right),
\end{aligned}
$$

where $\beta=\frac{\alpha}{2 \alpha+2}$.

Analogously, we get from (34) that

$$
\begin{aligned}
& \mu_{0} \int_{D \backslash D_{\varepsilon}}\left(\gamma_{i}-\gamma_{e}\right) \nabla \tilde{v}_{\varepsilon}^{e} \cdot \nabla u_{0}^{i} d x \\
& =-\mu_{0}^{2} \varepsilon\left(\gamma_{i}-\gamma_{e}\right) \int_{\partial D \cap\{h<0\}} h\left[\left(\frac{\partial u_{0}^{i}}{\partial \tau}\right)^{2}+\frac{\gamma_{i}}{\gamma_{e}}\left(\frac{\partial u_{0}^{i}}{\partial \nu}\right)^{2}\right] d \sigma+O\left(\varepsilon^{1+\beta}\right) \\
& =-\mu_{0}^{2} \varepsilon\left(\gamma_{i}-\gamma_{e}\right) \int_{\partial D \cap\{h<0\}} h\left[\left(\frac{\partial u_{0}^{e}}{\partial \tau}\right)^{2}+\frac{\gamma_{e}}{\gamma_{i}}\left(\frac{\partial u_{0}^{e}}{\partial \nu}\right)^{2}\right] d \sigma+O\left(\varepsilon^{1+\beta}\right),
\end{aligned}
$$

where the last equality follows from the transmission conditions (4) and (5).

It now follows from (35) and (36) that for $\varepsilon$ small enough,

$$
\begin{aligned}
& \left\langle\left(T-T_{\varepsilon}\right) u_{0}, u_{0}\right\rangle \\
& =-\mu_{0}^{2} \varepsilon\left(\gamma_{i}-\gamma_{e}\right) \int_{\partial D} h\left[\left(\frac{\partial u_{0}^{e}}{\partial \tau}\right)^{2}+\frac{\gamma_{e}}{\gamma_{i}}\left(\frac{\partial u_{0}^{e}}{\partial \nu}\right)^{2}\right] d \sigma+O\left(\varepsilon^{1+\beta}\right) .
\end{aligned}
$$

In view of (10) and (26), we finally obtain

$$
\mu_{\varepsilon}-\mu_{0}=-\mu_{0}^{2} \varepsilon\left(\gamma_{i}-\gamma_{e}\right) \int_{\partial D} h\left[\left(\frac{\partial u_{0}^{e}}{\partial \tau}\right)^{2}+\frac{\gamma_{e}}{\gamma_{i}}\left(\frac{\partial u_{0}^{e}}{\partial \nu}\right)^{2}\right] d \sigma+O\left(\varepsilon^{1+\beta}\right)
$$

for some $\beta>0$. Since $\mu_{0}, \mu_{\varepsilon}$ are negative we can set $\mu_{0}^{-1}=-\omega_{0}^{2}$ and $\mu_{\varepsilon}^{-1}=-\omega_{\varepsilon}^{2}$. With this substitution and simple manipulations we get (7) and Theorem 2.1 is proved.

\section{ReCONSTRUCTION METHOD}

In order to reconstruct the perturbation $\varepsilon h$ from modal measurements, a first idea is to minimize the difference between the measured and the computed eigenvalues by using a least-square approach. This yields a laborious reconstruction algorithm which may not converge if we start away from the solution. Another idea, which sounds more attractive is to take advantage of the smallness of $\varepsilon$ and minimize over $\varepsilon h$ the quantity

$$
\left|\omega_{\varepsilon}^{2}-\omega_{0}^{2}+\left(\gamma_{i}-\gamma_{e}\right) \int_{\partial D} \varepsilon h(x)\left[\left(\frac{\partial u_{0}^{e}}{\partial \tau}(x)\right)^{2}+\frac{\gamma_{e}}{\gamma_{i}}\left(\frac{\partial u_{0}^{e}}{\partial \nu}(x)\right)^{2}\right] d \sigma_{x}\right| .
$$


A problem with this approach is that oscillations in $h$ cannot be determined effectively. This comes from the fact that the application

$$
h(x) \mapsto \int_{\partial D} h(x)\left[\left(\frac{\partial u_{0}^{e}}{\partial \tau}(x)\right)^{2}+\frac{\gamma_{e}}{\gamma_{i}}\left(\frac{\partial u_{0}^{e}}{\partial \nu}(x)\right)^{2}\right] d \sigma_{x}
$$

acts like a filter.

In this section, we rigorously establish a reconstruction formula for the function $h$ which allows us to determine $h$ with better resolution by less filtering of oscillations. Based on this dual formula, we then formulate the reconstruction of $h$ as an optimization problem.

3.1. Dual asymptotic formula. Let $u_{0}$ be the eigenfunction of (2). For $g \in$ $L^{2}(\partial \Omega)$ satisfying $\int_{\partial \Omega} g u_{0}=0$, let $w_{g}$ be the solution to

$$
\left\{\begin{aligned}
\nabla \cdot\left(\gamma_{D} \nabla w_{g}\right) & =-\omega_{0}^{2} w_{g} \quad \text { in } \quad \Omega, \\
\gamma_{D} \frac{\partial w_{g}}{\partial \nu} & =g \quad \text { on } \quad \partial \Omega, \\
\int_{\Omega} w_{g} u_{0} & =1 .
\end{aligned}\right.
$$

Multiplying the first equation in (38) by $u_{\varepsilon}$ and integrating over $\Omega$, we get from the divergence theorem

$$
\int_{\partial \Omega} g u_{\varepsilon}+\omega_{0}^{2} \int_{\Omega} w_{g} u_{\varepsilon}=\int_{\Omega} \gamma_{D} \nabla u_{\varepsilon} \cdot \nabla w_{g}
$$

Since $\int_{\partial \Omega} g u_{0}=0$ and

$$
\omega_{\varepsilon}^{2} \int_{\Omega} w_{g} u_{\varepsilon}=\int_{\Omega} \gamma_{D_{\varepsilon}} \nabla u_{\varepsilon} \cdot \nabla w_{g}
$$

we obtain

$$
\int_{\partial \Omega} g\left(u_{\varepsilon}-u_{0}\right)+\left(\omega_{0}^{2}-\omega_{\varepsilon}^{2}\right) \int_{\Omega} w_{g} u_{\varepsilon}=-\int_{\Omega}\left(\gamma_{D_{\varepsilon}}-\gamma_{D}\right) \nabla u_{\varepsilon} \cdot \nabla w_{g} d x .
$$

By Theorem 2.1 we have that for $\varepsilon$ small enough, $\omega_{0}^{2}-\omega_{\varepsilon}^{2}=O(\varepsilon)$. Furthermore, since $u_{\varepsilon} \rightarrow u_{0}$ in $L^{2}(\Omega)$ as $\varepsilon \rightarrow 0$, we derive

$$
\int_{\partial \Omega} g\left(u_{\varepsilon}-u_{0}\right)+\left(\omega_{0}^{2}-\omega_{\varepsilon}^{2}\right) \int_{\Omega} w_{g} u_{0}=-\int_{\Omega}\left(\gamma_{D_{\varepsilon}}-\gamma_{D}\right) \nabla u_{\varepsilon} \cdot \nabla w_{g} d x+O\left(\varepsilon^{1+\beta}\right),
$$

for some $\beta>0$.

We now prove the following theorem in the same way as in the previous section. The asymptotic formula in the theorem can be regarded as a dual formula to that of $\omega_{\varepsilon}^{2}-\omega_{0}^{2}$. It plays a key role in our reconstruction procedure.

Theorem 3.1. The following asymptotic formula holds as $\varepsilon \rightarrow 0$ :

$$
\begin{aligned}
& \int_{\partial \Omega} g\left(u_{\varepsilon}-u_{0}\right)+\left(\omega_{0}^{2}-\omega_{\varepsilon}^{2}\right) \int_{\Omega} w_{g} u_{0} \\
& =\varepsilon\left(\gamma_{i}-\gamma_{e}\right) \int_{\partial D} h(x)\left(\frac{\partial u_{0}^{e}}{\partial \tau}(x) \frac{\partial w_{g}^{e}}{\partial \tau}(x)+\frac{\gamma_{e}}{\gamma_{i}} \frac{\partial u_{0}^{e}}{\partial \nu}(x) \frac{\partial w_{g}^{e}}{\partial \nu}(x)\right) d \sigma_{x}+O\left(\varepsilon^{1+\beta}\right)
\end{aligned}
$$

for some $\beta>0$. 
Proof. In view of (39), it suffices to show that

$$
\begin{aligned}
& \int_{\Omega}\left(\gamma_{D_{\varepsilon}}-\gamma_{D}\right) \nabla u_{\varepsilon} \cdot \nabla w_{g} \\
& =-\left(\gamma_{i}-\gamma_{e}\right) \varepsilon \int_{\partial D} h(x)\left(\frac{\partial u_{0}^{e}}{\partial \tau}(x) \frac{\partial w_{g}^{e}}{\partial \tau}(x)+\frac{\gamma_{e}}{\gamma_{i}} \frac{\partial u_{0}^{e}}{\partial \nu}(x) \frac{\partial w_{g}^{e}}{\partial \nu}(x)\right) d \sigma_{x} \\
& \quad+O\left(\varepsilon^{1+\beta}\right) .
\end{aligned}
$$

To prove the lemma we use the gradient estimates for $u_{\varepsilon}$ (see (23) and (24)). We can show that the same kind of estimates hold for $w_{g}$ :

$$
\left\|w_{g}\right\|_{\mathcal{C}^{1, \alpha}(\bar{D})} \leq C
$$

and

$$
\left\|w_{g}\right\|_{\mathcal{C}^{1, \alpha}\left(\Omega_{d_{0} / 2} \backslash D\right)} \leq C .
$$

Noting that $\left(w_{g}-u_{0}\right)$ belongs to the subspace of $H^{1}(\Omega)$ orthogonal to $u_{0}$, these estimates follow immediately from [16] since on one hand, the operator $\nabla \cdot \gamma_{D} \nabla+\omega_{0}^{2}$ in $\Omega$ with Neumann boundary conditions on $\partial \Omega$ is well-posed on the subspace of $H^{1}(\Omega)$ orthogonal to $u_{0}$, and on the other hand, $u_{0}$ itself satisfies such estimates.

Proceeding similarly as we did for estimating $\int_{D_{\varepsilon} \backslash D} \nabla \tilde{v}_{\varepsilon}^{i} \cdot \nabla u_{0}^{e} d x$ in the previous section, we have

$$
\begin{aligned}
& \int_{\Omega}\left(\gamma_{D_{\varepsilon}}-\gamma_{D}\right) \nabla u_{\varepsilon} \cdot \nabla w_{g} \\
& \quad=\int_{D_{\varepsilon} \backslash D}\left(\gamma_{i}-\gamma_{e}\right) \nabla u_{\varepsilon}^{i} \cdot \nabla w_{g}^{e}-\int_{D \backslash D_{\varepsilon}}\left(\gamma_{i}-\gamma_{e}\right) \nabla u_{\varepsilon}^{e} \cdot \nabla w_{g}^{i} .
\end{aligned}
$$

Changing variables and using the gradient estimates for $u_{\varepsilon}$ and $w_{g}$ we obtain

$$
\begin{aligned}
\int_{D_{\varepsilon} \backslash D} & \left(\gamma_{i}-\gamma_{e}\right) \nabla u_{\varepsilon}^{i} \cdot \nabla w_{g}^{e} d x \\
= & \int_{0}^{\varepsilon} \int_{\partial D \cap\{h>0\}} h(x)\left(\gamma_{i}-\gamma_{e}\right) \nabla u_{\varepsilon}^{i}\left(x_{\varepsilon}\right) \cdot \nabla w_{g}^{e}\left(x_{\varepsilon}\right) d \sigma_{x} d t+O\left(\varepsilon^{1+\beta}\right),
\end{aligned}
$$

and analogously

$$
\begin{aligned}
\int_{D \backslash D_{\varepsilon}} & \left(\gamma_{i}-\gamma_{e}\right) \nabla u_{\varepsilon}^{e} \cdot \nabla w_{g}^{i} d x \\
= & \int_{0}^{\varepsilon} \int_{\partial D \cap\{h<0\}}(-h(x))\left(\gamma_{i}-\gamma_{e}\right) \nabla u_{\varepsilon}^{e}\left(x_{\varepsilon}\right) \cdot \nabla w_{g}^{i}\left(x_{\varepsilon}\right) d \sigma_{x} d t+O\left(\varepsilon^{1+\beta}\right) .
\end{aligned}
$$

Using the transmission conditions, we get

$$
\begin{aligned}
& \int_{D_{\varepsilon} \backslash D}\left(\gamma_{i}-\gamma_{e}\right) \nabla u_{\varepsilon}^{i} \cdot \nabla w_{g}^{e} d x \\
& =\varepsilon \int_{\partial D \cap\{h>0\}} h(x)\left(\gamma_{i}-\gamma_{e}\right)\left(\frac{\partial u_{\varepsilon}^{e}}{\partial \tau}\left(x_{\varepsilon}\right) \frac{\partial w_{g}^{e}}{\partial \tau}\left(x_{\varepsilon}\right)+\frac{\gamma_{e}}{\gamma_{i}} \frac{\partial u_{\varepsilon}^{e}}{\partial \nu}\left(x_{\varepsilon}\right) \frac{\partial w_{g}^{e}}{\partial \nu}\left(x_{\varepsilon}\right)\right) d \sigma_{x} \\
& \quad+O\left(\varepsilon^{1+\beta}\right)
\end{aligned}
$$


and

$$
\begin{aligned}
& \int_{D \backslash D_{\varepsilon}}\left(\gamma_{i}-\gamma_{e}\right) \nabla u_{\varepsilon}^{e} \cdot \nabla w_{g}^{i} d x \\
& =-\varepsilon \int_{\partial D \cap\{h<0\}} h(x)\left(\gamma_{i}-\gamma_{e}\right)\left(\frac{\partial u_{\varepsilon}^{i}}{\partial \tau}\left(x_{\varepsilon}\right) \frac{\partial w_{g}^{i}}{\partial \tau}\left(x_{\varepsilon}\right)+\frac{\gamma_{i}}{\gamma_{e}} \frac{\partial u_{\varepsilon}^{i}}{\partial \nu}\left(x_{\varepsilon}\right) \frac{\partial w_{g}^{i}}{\partial \nu}\left(x_{\varepsilon}\right)\right) d \sigma_{x} \\
& \quad+O\left(\varepsilon^{1+\beta}\right) .
\end{aligned}
$$

In order to replace $u_{\varepsilon}$ with $u_{0}$ in (43) and (44), we shall show that

$$
\left\|\nabla\left(u_{\varepsilon}^{e}-u_{0}^{e}\right)\right\|_{L^{\infty}\left(\partial D_{\varepsilon} \backslash D\right)}+\left\|\nabla\left(u_{\varepsilon}^{i}-u_{0}^{i}\right)\right\|_{L^{\infty}\left(\partial D_{\varepsilon} \cap \bar{D}\right)} \leq C \varepsilon^{\frac{\alpha}{2 \alpha+2}}
$$

for some constant $C$ independent of $\varepsilon$, following the same arguments as those in the proof of Lemma 2.2.

For doing so, let $2 \varepsilon<d<d_{0} / 2$ and let $\Omega_{d}^{\varepsilon}=\left\{x \in \Omega \backslash\left(D \cup D_{\varepsilon}\right): \operatorname{dist}(x, \partial(\Omega \backslash D \cup\right.$ $\left.\left.D_{\varepsilon}\right)>d\right\}$. Since $\theta_{\varepsilon}=\nabla\left(u_{\varepsilon}-u_{0}\right)$ is the solution of the following equation in $\Omega \backslash D \cup D_{\varepsilon}$,

$$
\Delta \theta_{\varepsilon}-\mu_{\varepsilon} \theta_{\varepsilon}=\left(\mu_{\varepsilon}-\mu_{0}\right) \nabla u_{0}
$$

then we may apply Theorem 8.17 of [11] to obtain

$$
\left\|\nabla\left(u_{\varepsilon}^{e}-u_{0}^{e}\right)\right\|_{L^{\infty}\left(\Omega_{d}^{\varepsilon}\right)} \leq C\left(\left\|\nabla\left(u_{\varepsilon}-u_{0}\right)\right\|_{L^{2}(\Omega)}+\left|\mu_{\varepsilon}-\mu_{0}\right|\left\|\nabla u_{0}\right\|_{L^{2}(\Omega)}\right) .
$$

Using the energy estimates and the fact that $\left|\mu_{\varepsilon}-\mu_{0}\right| \leq C \varepsilon$ we get

$$
\left\|\nabla\left(u_{\varepsilon}^{e}-u_{0}^{e}\right)\right\|_{L^{\infty}\left(\Omega_{d}^{\varepsilon}\right)} \leq \frac{C}{d} \sqrt{\varepsilon} .
$$

Now, let $y \in \partial D_{\varepsilon} \backslash D$ and let $y_{d}$ denote the closest point to $y$ in the set $\Omega_{d}^{\varepsilon}$. From (23) and (24) it follows that

$$
\left|\nabla u_{\varepsilon}^{e}(y)-\nabla u_{\varepsilon}^{e}\left(y_{d}\right)\right| \leq C d^{\alpha},
$$

which yields

$$
\begin{aligned}
\left|\nabla\left(u_{\varepsilon}^{e}-u_{0}^{e}\right)(y)\right| \leq & \left|\nabla u_{\varepsilon}^{e}(y)-\nabla u_{\varepsilon}^{e}\left(y_{d}\right)\right|+\left|\nabla u_{\varepsilon}^{e}\left(y_{d}\right)-\nabla u_{0}^{e}\left(y_{d}\right)\right| \\
& +\left|\nabla u_{0}^{e}\left(y_{d}\right)-\nabla u_{0}^{e}(y)\right| \\
\leq & C\left(d^{\alpha}+d^{-1} \varepsilon^{1 / 2}\right) .
\end{aligned}
$$

Here we also used the gradient estimates for $u_{0}$. By choosing $d=\varepsilon^{\frac{1}{2(\alpha+1)}}$ we get

$$
\left\|\nabla\left(u_{\varepsilon}^{e}-u_{0}^{e}\right)\right\|_{L^{\infty}\left(\partial D_{\varepsilon} \backslash D\right)} \leq C \varepsilon^{\frac{\alpha}{2 \alpha+2}} .
$$

In a similar way one can prove that

$$
\left\|\nabla\left(u_{\varepsilon}^{i}-u_{0}^{i}\right)\right\|_{L^{\infty}\left(\partial D_{\varepsilon} \cap \bar{D}\right)} \leq C \varepsilon^{\frac{\alpha}{2 \alpha+2}} .
$$

Finally, inserting this into (43) and (44) and using the gradient estimates for $u_{0}$ we get

$$
\begin{aligned}
& \int_{D_{\varepsilon} \backslash D}\left(\gamma_{i}-\gamma_{e}\right) \nabla u_{\varepsilon}^{i} \cdot \nabla w_{g}^{e} d x \\
& =\varepsilon \int_{\partial D \cap\{h>0\}} h(x)\left(\gamma_{i}-\gamma_{e}\right)\left(\frac{\partial u_{0}^{e}}{\partial \tau}(x) \frac{\partial w_{g}^{e}}{\partial \tau}(x)+\frac{\gamma_{e}}{\gamma_{i}} \frac{\partial u_{0}^{e}}{\partial \nu}(x) \frac{\partial w_{g}^{e}}{\partial \nu}(x)\right) d \sigma_{x}+O\left(\varepsilon^{1+\beta}\right)
\end{aligned}
$$


and

$$
\begin{aligned}
& \int_{D \backslash D_{\varepsilon}}\left(\gamma_{i}-\gamma_{e}\right) \nabla u_{\varepsilon}^{e} \cdot \nabla w_{g}^{i} d x \\
& =-\varepsilon \int_{\partial D \cap\{h<0\}} h(x)\left(\gamma_{i}-\gamma_{e}\right)\left(\frac{\partial u_{0}^{i}}{\partial \tau}(x) \frac{\partial w_{g}^{i}}{\partial \tau}(x)+\frac{\gamma_{i}}{\gamma_{e}} \frac{\partial u_{0}^{i}}{\partial \nu}(x) \frac{\partial w_{g}^{i}}{\partial \nu}(x)\right) d \sigma_{x}+O\left(\varepsilon^{1+\beta}\right) .
\end{aligned}
$$

Applying the transmission conditions (44) and (5) once more, summing up the two integrals and inserting the sum into (39) we obtain (40). This completes the proof.

3.2. Optimization problem. In view of Theorem 3.1, the reconstruction method is rather apparent. With the measurements $\left(\omega_{\varepsilon}^{2}-\omega_{0}^{2},\left.\left(u_{\varepsilon}-u_{0}\right)\right|_{\partial \Omega}\right)$ and a finite number of linearly independent functions $g_{1}, \ldots, g_{L}$ on $\partial \Omega$ satisfying $\int_{\partial \Omega} g_{l} u_{0} d \sigma=$ 0 , define the functional $J(h)$ by

$$
\begin{aligned}
J(h) & :=\sum_{l=1}^{L} \mid \int_{\partial \Omega} g_{l}\left(u_{\varepsilon}-u_{0}\right)+\left(\omega_{0}^{2}-\omega_{\varepsilon}^{2}\right) \int_{\Omega} w_{g_{l}} u_{0} \\
& -\left.\varepsilon \int_{\partial D} h(x)\left(\gamma_{i}-\gamma_{e}\right)\left(\frac{\partial u_{0}^{e}}{\partial \tau}(x) \frac{\partial w_{g_{l}}^{e}}{\partial \tau}(x)+\frac{\gamma_{e}}{\gamma_{i}} \frac{\partial u_{0}^{e}}{\partial \nu}(x) \frac{\partial w_{g_{l}}^{e}}{\partial \nu}(x)\right) d \sigma_{x}\right|^{2} .
\end{aligned}
$$

The method for reconstructing the shape deformation is to minimize $J(h)$ over $h$. If the small parameter $\varepsilon$ is known, then by minimizing $J(h)$ over $h$ we can reconstruct $h$. If $\varepsilon$ is unknown, we may consider the functional $J$ as a function of $\varepsilon h$ instead of $h$ to obtain the deformation $\varepsilon h$.

It is worth emphasizing that the integral

$$
\varepsilon \int_{\partial D} h(x)\left(\frac{\partial u_{0}^{e}}{\partial \tau}(x) \frac{\partial w_{g}^{e}}{\partial \tau}(x)+\frac{\gamma_{e}}{\gamma_{i}} \frac{\partial u_{0}^{e}}{\partial \nu}(x) \frac{\partial w_{g}^{e}}{\partial \nu}(x)\right) d \sigma_{x}
$$

filters less oscillations in $h$ than the one in (37) because of the flexibility we have in choosing $w_{g}$ and therefore, minimizing $J(h)$ allows better reconstruction of $h$.

The best choice of $g_{1}, \ldots, g_{L}$ is such that the functions

$$
v_{g}=\frac{\partial u_{0}^{e}}{\partial \tau} \frac{\partial w_{g}^{e}}{\partial \tau}+\frac{\gamma_{e}}{\gamma_{i}} \frac{\partial u_{0}^{e}}{\partial \nu} \frac{\partial w_{g}^{e}}{\partial \nu} \quad \text { for } g=g_{1}, \ldots, g_{L}
$$

on $\partial D$ are highly oscillating. To formalize this, introduce the operator $\Lambda$ defined for $g \in V(\partial \Omega)=\left\{g \in L^{2}(\partial \Omega): \int_{\partial \Omega} g u_{0}=0\right\}$ by $\Lambda(g)=\left.w_{g}\right|_{\partial D}$, where $w_{g}$ is the solution to (38). The best choice is then to take $\left\{g_{1}, \ldots, g_{L}\right\}$ as a basis of the image space of $\Lambda^{*} \Lambda$, where $\Lambda^{*}: L^{2}(\partial D) \rightarrow V(\partial \Omega)$ is the adjoint of $\Lambda$. Indeed, the only changes that we can reconstruct are linear combinations of $\left.v_{g}\right|_{\partial D}$ for $g \in \operatorname{Image}\left(\Lambda^{*} \Lambda\right)$. Indeed, the reconstruction is robust with respect to noise in measurements and errors in the a priori knowledge of the reference inclusion. For this, see [3].

Nevertheless, since computing Image $\left(\Lambda^{*} \Lambda\right)$ is costly, we just take our numerical examples $\Omega$ and $D$ to be disks and the functions $g_{1}, \ldots, g_{L}$ to be cosine and sine functions. Note that if there is an index $i$ for which the $i$ th Fourier coefficients of $\left.v_{g_{l}}\right|_{\partial D}, l=1, \ldots, L$ are all zero, then the $i$ th Fourier coefficient of $h$ cannot be reconstructed by our algorithm. 


\section{The CASE of A MUltiple eigenvalue}

In this section we consider the case of a multiple eigenvalue. We first derive an averaged approximation formula for a multiple eigenvalue. This is based on a standard argument from [17, 13] that the mean of the cluster resulting from the eigenvalue splitting of converging eigenvalues better approximates the limit eigenvalue than any of the individual eigenvalues from the cluster. Then we provide a reconstruction formula for the interface changes that has the same general form as the one in the simple eigenvalue case.

Let $\omega_{0}^{2}$ denote an eigenvalue of the problem for (2) with geometric multiplicity $m$ and let $\left\{u_{0, j}\right\}_{j=1, \ldots, m}$ be $L^{2}$-orthonormal eigenfunctions corresponding to $\omega_{0}^{2}$. Let $\left(\omega_{\varepsilon}^{j}\right)^{2}$ be the eigenvalues of problem (6) for $\varepsilon>0$ that are generated by splitting from $\omega_{0}^{2}$ and let $u_{\varepsilon}^{j}$ be the associated eigenfunction (normalized with respect to $L^{2}$ ) such that $u_{\varepsilon}^{j} \rightarrow u_{0, j}$ as $\varepsilon \rightarrow 0$.

Then, by (8) and (9) and proceeding similarly as in the proof of Theorem 2.1] we get the following result.

Theorem 4.1. As $\varepsilon \rightarrow 0$, the following asymptotic expansion holds:

$$
\begin{aligned}
& \frac{1}{\omega_{0}^{2}}-\frac{1}{m} \sum_{j=1}^{m} \frac{1}{\left(\omega_{\varepsilon}^{j}\right)^{2}} \\
& =-\frac{\varepsilon\left(\gamma_{i}-\gamma_{e}\right)}{m \omega_{0}^{4}} \sum_{j=1}^{m} \int_{\partial D} h(x)\left(\left(\frac{\partial u_{0, j}^{e}}{\partial \tau}(x)\right)^{2}+\frac{\gamma_{e}}{\gamma_{i}}\left(\frac{\partial u_{0, j}^{e}}{\partial \nu}(x)\right)^{2}\right) d \sigma_{x}+O\left(\varepsilon^{1+\beta}\right),
\end{aligned}
$$

for some $\beta>0$.

Using Theorem 4.1, we can adapt the algorithm described in the previous section to reconstruct the shape deformation in the case of a multiple eigenvalue. For $g \in L^{2}(\partial \Omega)$ satisfying $\int_{\partial \Omega} g u_{0, j}=0$ for $j=1, \ldots, m$, let $w_{g}$ be the solution to

$$
\left\{\begin{aligned}
\nabla \cdot\left(\gamma_{D} \nabla w_{g}\right) & =-\omega_{0}^{2} w_{g} \quad \text { in } \Omega \\
\gamma_{D} \frac{\partial w_{g}}{\partial \nu} & =g \quad \text { on } \partial \Omega \\
\int_{\Omega} w_{g} u_{0, j} & =1, \quad j=1, \ldots, m .
\end{aligned}\right.
$$

The method for reconstructing the shape deformation in the case of a multiple eigenvalue $\omega_{0}^{2}$ is to minimize the functional $J(h)$ over $h$ where $J$ is given by

$$
\begin{aligned}
J(h) & :=\sum_{l=1}^{L} \mid \frac{1}{m} \sum_{j=1}^{m} \int_{\partial \Omega} g_{l}\left(u_{\varepsilon}^{j}-u_{0, j}\right)-\frac{1}{m} \sum_{j=1}^{m}\left(\omega_{\varepsilon}^{j}\right)^{2}+\omega_{0}^{2} \\
& -\left.\frac{\varepsilon}{m} \sum_{j=1}^{m} \int_{\partial D} h(x)\left(\gamma_{i}-\gamma_{e}\right)\left(\frac{\partial u_{0, j}^{e}}{\partial \tau}(x) \frac{\partial w_{g_{l}}^{e}}{\partial \tau}(x)+\frac{\gamma_{e}}{\gamma_{i}} \frac{\partial u_{0, j}^{e}}{\partial \nu}(x) \frac{\partial w_{g_{l}}^{e}}{\partial \nu}(x)\right) d \sigma_{x}\right|^{2}
\end{aligned}
$$

where $g_{1}, \ldots, g_{L}$ are linearly independent functions.

\section{NumericAl EXAMPLES}

We now present numerical examples of the shape deformation reconstruction method described in the previous section. In the following examples, the background domain $\Omega$ is assumed to be the unit disk centered at the origin and the 
(unperturbed) inclusion $D$ is the disk centered at $(0,-0.2)$ with radius 0.4 . We fix the conductivities:

$$
\gamma_{e}=1 \text { and } \gamma_{i}=1.5
$$

In order to acquire (simulated) data, we use a boundary integral method. Let $\mathcal{S}_{D}^{\omega}$ and $\mathcal{D}_{D}^{\omega}$ be the single and double layer potentials on $\partial D$ defined by the fundamental (outgoing) solution $\Gamma_{\omega}(x)=-\frac{i}{4} H_{0}^{(1)}(\omega|x|)$ to the operator $\Delta+\omega^{2}$ :

$$
\begin{aligned}
\mathcal{S}_{D}^{\omega}[\varphi](x) & =\int_{\partial D} \Gamma_{\omega}(x-y) \varphi(y) d \sigma(y), x \in \mathbb{R}^{2}, \\
\mathcal{D}_{D}^{\omega}[\varphi](x) & =\int_{\partial D} \frac{\partial \Gamma_{\omega}(x-y)}{\partial \nu_{y}} \varphi(y) d \sigma(y), x \in \mathbb{R}^{2} \backslash \partial D,
\end{aligned}
$$

for $\varphi \in L^{2}(\partial D)$. Here $H_{0}^{(1)}$ is the Hankel function of the first kind and order 0 .

We define analogously $\mathcal{S}_{\Omega}^{\omega}$ and $\mathcal{D}_{\Omega}^{\omega}$ to be the single and double layer potentials on $\partial \Omega$.

Given the scalar $\omega$ the solution $u_{0}$ to (2) can be represented as

$$
u_{0}(x)= \begin{cases}\mathcal{S}_{D}^{\frac{\omega}{\sqrt{\gamma_{i}}}}[\varphi](x), & x \in D, \\ \mathcal{S}_{D}^{\omega}[\psi](x)+\mathcal{D}_{\Omega}^{\omega}[f](x), & x \in \Omega \backslash \bar{D},\end{cases}
$$

where $f=\left.u_{0}\right|_{\partial \Omega}$ and $(\varphi, \psi) \in L^{2}(\partial D) \times L^{2}(\partial D)$ is a solution to the following integral equation:

$$
\begin{cases}\mathcal{S}_{D}^{\frac{\omega}{\sqrt{\gamma_{i}}}}[\varphi]=\mathcal{S}_{D}^{\omega}[\psi]+\mathcal{D}_{\Omega}^{\omega}[f] & \text { on } \partial D, \\ \gamma_{i} \frac{\partial}{\partial \nu}\left(\mathcal{S}_{D}^{\frac{\omega}{\sqrt{\gamma_{i}}}}[\varphi]\right)^{i}=\frac{\partial}{\partial \nu}\left(\mathcal{S}_{D}^{\omega}[\psi]+\mathcal{D}_{\Omega}^{\omega}[f]\right)^{e} & \text { on } \partial D \\ \mathcal{S}_{D}^{\omega}[\psi]+\left(\mathcal{D}_{\Omega}^{\omega}[f]\right)^{i}=f & \text { on } \partial \Omega\end{cases}
$$

Note that $\omega=\omega_{0}$ is an eigenvalue of (2) if and only if (49) has a nontrivial solution.

The computations of the eigenvalues and the eigenfunctions on the boundary $\partial \Omega$ are performed numerically with an accuracy much higher than $\epsilon$ because otherwise this would affect dramatically the reconstruction algorithm by making the reconstruction of $h$ inaccurate.

The function $w_{g_{l}}$ is calculated for

$$
g_{l}=a_{l}+b_{l} \cos \theta+c_{l} \sin (l+1) \theta+d_{l} \cos (l+1) \theta, 1 \leq l \leq L(=8),
$$

where $a_{l}, b_{l}, c_{l}, d_{l}$ are constants chosen for $g_{l}$ to satisfy the normalization condition in (47). Again, problem (47) has to be solved very accurately in order to insure a quality of the solution to the auxiliary problem (47) enough to have an accurate reconstruction of $h$.

We simulate the reconstruction method for the perturbation function $h$ given by

$$
h(\theta)=1-2 \sin (j \theta), j=0,3,6,9, \text { and } \epsilon=0.02,0.04 .
$$

As $j$ increases, the oscillations in $h$ become higher and the reconstruction problem more difficult to solve.

In the reconstruction algorithm $h$ is approximated as follows:

$$
h(\theta) \approx h_{0}+\sum_{p=1}^{9}\left(h_{2 p-1} \cos p \theta+h_{2 p} \sin p \theta\right)=: \sum_{p=0}^{18} h_{p} \Phi_{p}(\theta),
$$


where

$$
\Phi_{0}(\theta)=1, \Phi_{2 p-1}(\theta)=\cos p \theta, \Phi_{2 p}(\theta)=\sin p \theta, \quad p=1, \ldots, 9 .
$$

For $1 \leq j \leq 2,1 \leq l \leq 8$, and $0 \leq p \leq 18$, define a matrix $M_{s}$ as

$$
\begin{aligned}
& M_{s}(2(j-1)+l, p) \\
& \quad:=\varepsilon(k-1) \int_{\partial D} \Phi_{p}(x)\left(\frac{\partial u_{0, j}^{e}}{\partial \tau}(x) \frac{\partial w_{g_{l}}^{e}}{\partial \tau}(x)+\frac{1}{k} \frac{\partial u_{0, j}^{e}}{\partial \nu}(x) \frac{\partial w_{g_{l}}^{e}}{\partial \nu}(x)\right) d \sigma_{x},
\end{aligned}
$$

where $s=1$ and $s=2$, respectively, stand for the first and third eigenvalues. Similarly, let the measurement vector $B_{s}$ for $s=1,2$ be defined by

$$
B_{s}(2(j-1)+l)=\int_{\partial \Omega} g_{l}\left(u_{\varepsilon}^{j}-u_{0, j}\right) d \sigma_{x}-\left(\left(\omega_{\epsilon}^{j}\right)^{2}-\omega_{0}^{2}\right) .
$$

For $s=1,2$, we obtain the coefficients $h_{p}^{s}$ using the formula

$$
\left(h_{0}^{s}, \ldots, h_{18}^{s}\right)=\left(M_{s}^{T} M_{s}+\delta I_{19}\right)^{-1} M_{s}^{T} B_{s},
$$

where $I_{19}$ is the $19 \times 19$ identity matrix and the regularization parameter $\delta$ is one of the following numbers $1,0.1,0.01,0.001$.

Now, to combine the first and third eigenvalue and eigenvector measurements we stack the two matrices $M_{1}$ and $M_{2}$ and the vectors $B_{1}$ and $B_{2}$ vertically, and compute new coefficients $h_{p}^{3}$ for $h$ by

$$
\begin{aligned}
& \left(h_{0}^{3}, \ldots, h_{18}^{3}\right) \\
& \quad=\left(\operatorname{Stack}\left(M_{1}, M_{2}\right)^{T} \operatorname{Stack}\left(M_{1}, M_{2}\right)+\delta I_{19}\right)^{-1} \operatorname{Stack}\left(M_{1}, M_{2}\right)^{T} \operatorname{Stack}\left(B_{1}, B_{2}\right) .
\end{aligned}
$$

Note that a similar formula could be derived if the eigenvalue is more than double.

Example 1. In this example, $h(\theta)=1-2 \sin (j \theta), j=0,3,6,9$, and $\epsilon=0.02$. Here and in the following examples, we assume that $\varepsilon$ is known and reconstruct $h$. In Figure 1, $h$ is approximated from the data corresponding to the first eigenvalue in the first column, and second eigenvalue in the second column, and first and second eigenvalues in the third column. The regularization parameter $\delta$ is taken to be equal to 0.001 except for $j=3$ and 6 on the third column. In these two exceptional cases, $\delta$ is chosen to be 0.1 . Figure 1 shows that first eigenvalue measurements work very well for not highly oscillating perturbations $h$, but it is not enough for higher oscillatory perturbation. This clearly indicates the resolution limit of our algorithm and shows that it is a function of the modal measurements we use. However, the quality of image is increased when second eigenvalue measurements are used as well.

Example 2. In this example, $h(\theta)=1-2 \sin (j \theta), j=0,3,6,9$, and $\epsilon=0.04$. Regularization parameter $\delta$ is 1 for all cases in this example. Reconstruction results are shown in Figure 2

Example 3. The example in Figure 3 shows the reconstruction of an inclusion which is shifted 0.2 to the right. First eigenvalue measurements are used, and regularization parameter $\delta$ is 0.01 . In this example, the obtained image is very close to the real one. 
Example 4. The example in Figure 4 shows the reconstruction of an inclusion which is perturbed and shifted to the right, i.e., $D$ is perturbed as $\epsilon h(\theta)=0.02(1-$ $2 \sin 6 \theta)$ and then shifted 0.2 to the right. Regularization parameter $\delta$ is 1 . In this example we used the first and third eigenvalues.
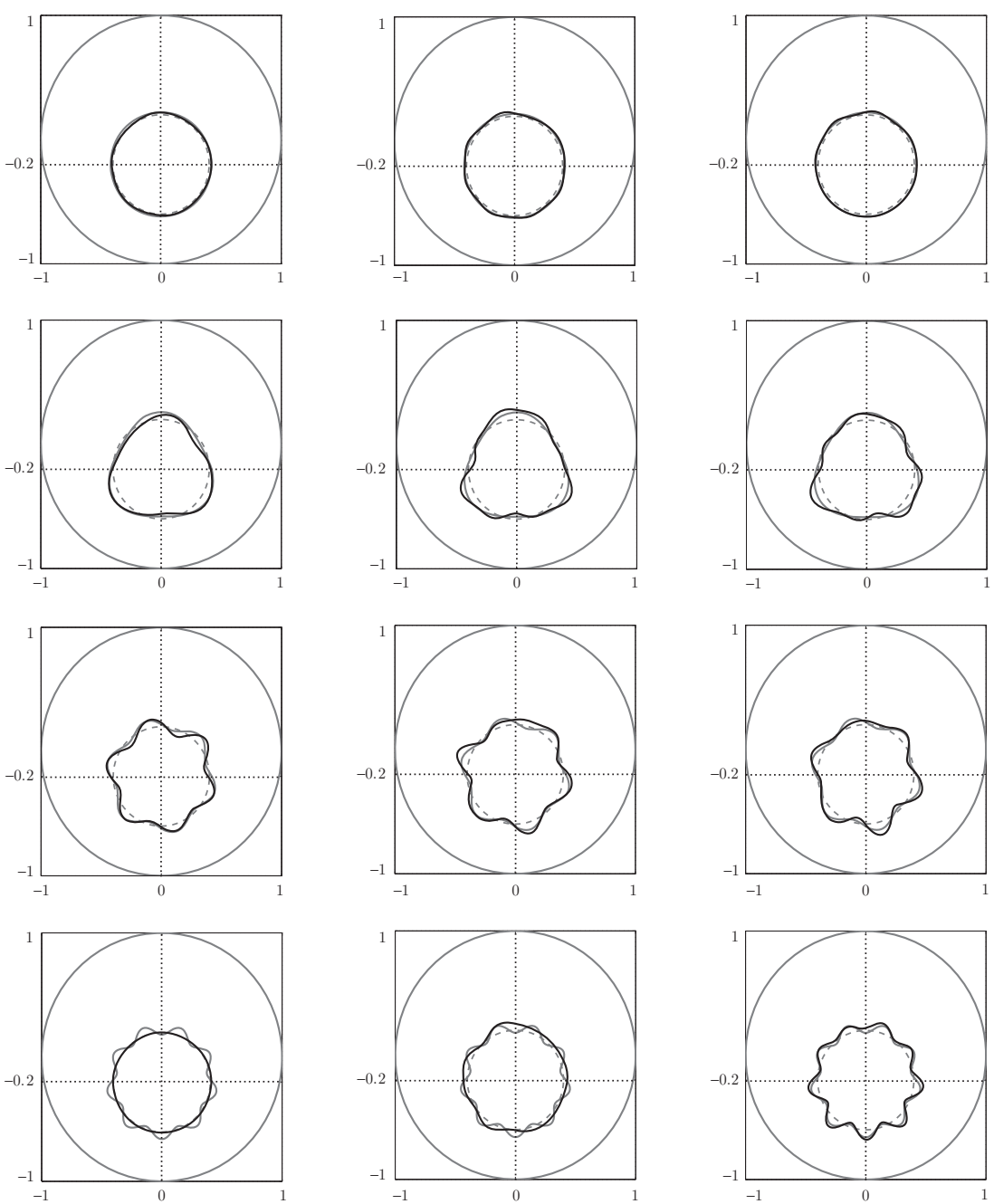

Figure 1. The solid whitest curves represent the inclusions, which are perturbations of disks, given by the dashed curves. The darkest curves are the reconstructed inclusions. The perturbation is given by $\epsilon h$ where $\epsilon=0.02$. 

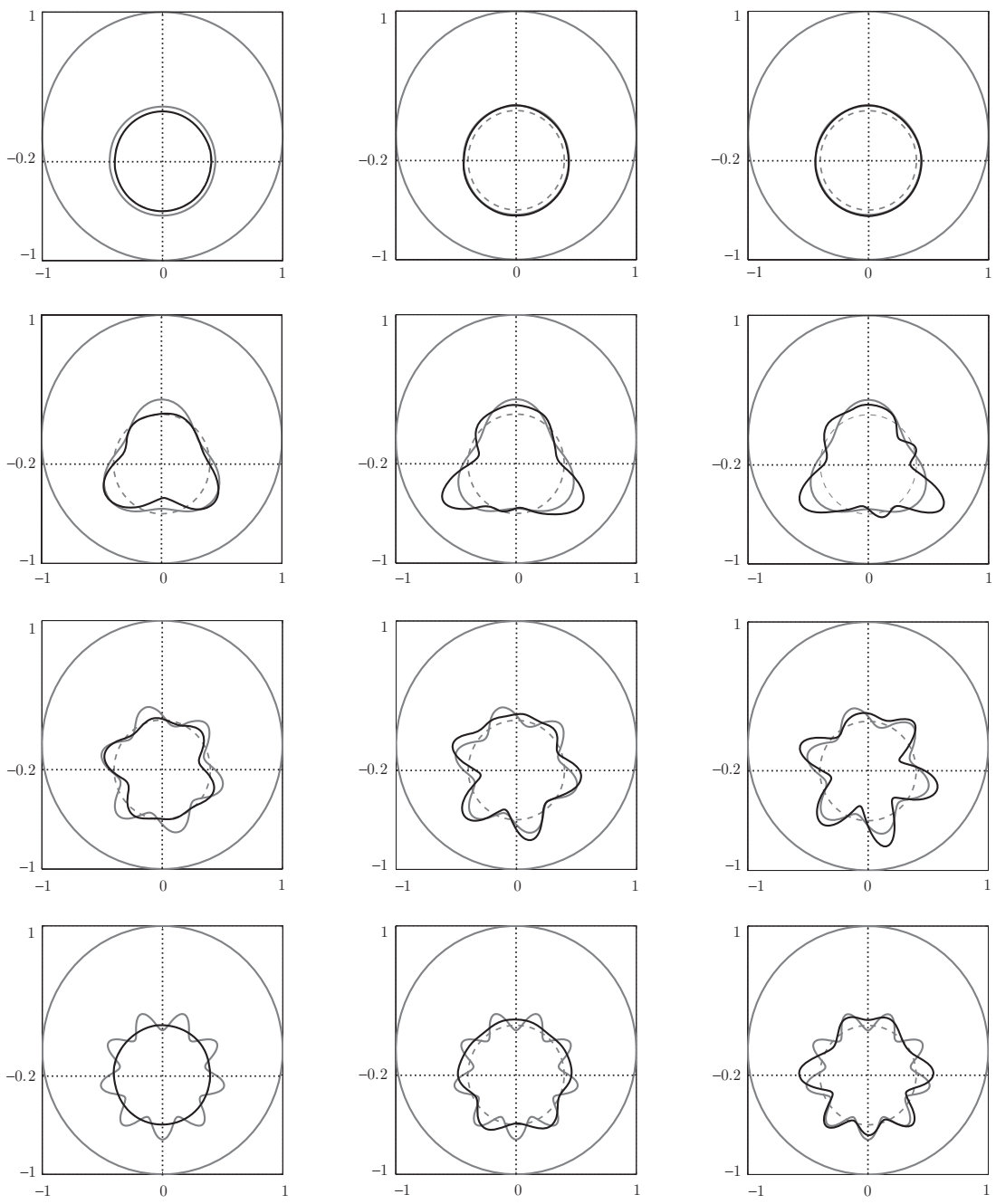

Figure 2. Reconstruction result when $\epsilon=0.04$.

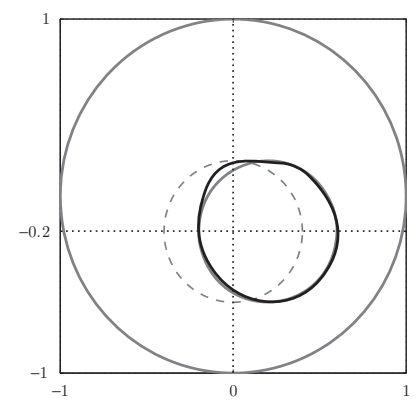

FiguRE 3. Reconstruction of a shifted inclusion. 

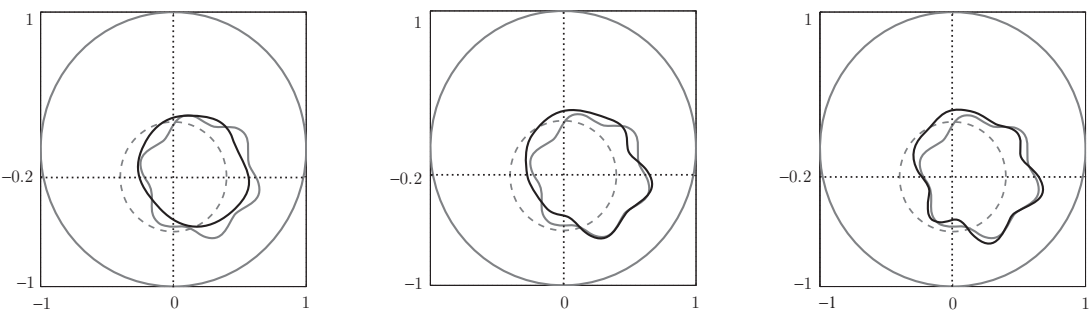

Figure 4. Reconstruction of a perturbed and shifted inclusion using the first eigenvalue (on the left), the third eigenvalue (in the middle) and both the first and the third eigenvalues (on the right).

\section{CONCLUding REMARKS}

In this paper we have introduced an optimization procedure for reconstructing interface changes of an inclusion from modal measurements. Our procedure takes advantage of the smallness of the changes. It is based on the dual asymptotic formula in Theorem 3.1. We have presented numerical experiments that show that our reconstruction procedure from eigenvalue measurements works pretty well for reconstructing perturbations of the interface. We have also pointed out the resolution limit of our procedure and observed how it increases as the used eigenfrequency increases. Indeed, we have showed that multi-modal measurements yield better reconstruction than those obtained by only one pair of modal parameters. Very recently, all of the results of this paper have been extended to linear elasticity in [1].

To conclude this paper, we make a few remarks. We first note that Theorem 2.1 may be used to compute the shape derivative of objective functionals involving eigenvalues of (2). Recall that if we consider the perturbation under the map $\theta$, then

$$
D_{\theta}=\{x+\theta(x): x \in D\},
$$

where $\theta \in W^{1, \infty}\left(\mathbb{R}^{2}, \mathbb{R}^{2}\right)$ is such that $\|\theta\|_{W^{1, \infty}}<1$, then the shape derivative of an objective functional $J(D)$ at $D$ is defined as the Fréchet differential of $\theta \mapsto J\left(D_{\theta}\right)$ at 0 , which depends only on $\theta \cdot \nu$ on the boundary $\partial D$.

Indeed, based again on Theorem 2.1 the level set approach developed by Osher and Santosa in [18] for solving the acoustic drum problem can be immediately generalized to the inclusion problem $\inf _{D} J(D)$. See, for instance, [5, 3].

Finally, it would be interesting to study the limit of (7) as $\gamma_{e}$ tends to 0 . In this case, $\omega_{\varepsilon}^{2} / \gamma_{i}$ and $\omega_{0}^{2} / \gamma_{i}$ approach the Neumann eigenvalues for $D_{\varepsilon}$ and $D$, respectively. Recall that the formula for the Neumann eigenvalue perturbation due to small deformation of the boundary is well known and the leading-order term is given by

$$
\varepsilon\left(\int_{\partial D} h\left|\nabla v_{0}\right|^{2} d \sigma-\omega_{0}^{2} \int_{\partial D} h\left|v_{0}\right|^{2} d \sigma\right),
$$

where $v_{0}$ is the (normalized) Neumann eigenfunction. See, for example, Section 5.6 in 20]. It would be interesting to show rigorously that the first-order term of the expansion of $\omega_{\varepsilon}^{2} / \gamma_{i}-\omega_{0}^{2} / \gamma_{i}$ in (77) converges to the one in (51) as $\gamma_{e} \rightarrow 0$. 


\section{ACKNOWLEDGMENTS}

The authors are very grateful to the referees for their very careful reading of a first version of this paper and their comments and suggestions. The partial support of the ANR projects EchoScan (AN-06-Blan-0089) and SISTAE (TLOG-004), the KOSEF grant R01-2006-000-10002-0, the MIUR, PRIN, grant n. 2006014115, and the STAR project 190117RD and K2-08-03-00-0001-08-B13-00-001-0-0 (KICOS) are also acknowledged.

\section{REFERENCES}

[1] H. Ammari, E. Beretta, E. Francini, H. Kang, and M. Lim, Reconstruction of small interface changes of an inclusion from modal measurements II: The elastic case, J. Math. Pures Appl., to appear.

[2] H. Ammari, E. Bonnetier, Y. Capdeboscq, M. Tanter, and M. Fink, Electrical impedance tomography by elastic deformation, SIAM J. Appl. Math., 68 (2008), 1557-1573. MR2424952 (2009h:35439)

[3] H. Ammari, P. Garapon, F. Jouve, and H. Kang, A new optimal control approach toward reconstruction of extended inclusions, preprint.

[4] H. Ammari, H. Kang, E. Kim, and H. Lee, Vibration testing for anomaly detection, Math. Meth. Appl. Sci., 32 (2009), 863-874. MR2507936

[5] H. Ammari, H. Kang, and H. Lee, Layer Potential Techniques in Spectral Analysis, Mathematical Surveys and Monographs, Vol. 153, American Math. Soc., Providence, 2009. MR2488135

[6] H. Ammari, H. Kang, M. Lim, and H. Zribi, Layer potential techniques in spectral analysis. Part I: Complete asymptotic expansions for eigenvalues of the Laplacian in domains with small inclusions, Trans. Amer. Math. Soc., to appear.

[7] H. Ammari, H. Kang, M. Lim, and H. Zribi, Conductivity interface problems. Part I: small perturbations of an interface, Trans. Amer. Math. Soc., to appear.

[8] H. Ammari and S. Moskow, Asymptotic expansions for eigenvalues in the presence of small inhomogeneities, Math. Meth. Appl. Sci., 26 (2003), 67-75. MR.1943110(2003j:35035)

[9] Y. Capdeboscq and M.S. Vogelius, A general representation formula for boundary voltage perturbations caused by internal conductivity inhomogeneities of low volume fraction, M2AN, 37 (2003), 159-173. MR.1972656 (2004b:35334)

[10] P.R. Garabedian and M. Schiffer, Convexity of domain functionals, J. Anal. Math., 2 (1953), 281-368. MR0060117 (15:627a)

[11] D. Gilbarg and N. Trudinger, Elliptic Partial Differential Equations of Second Order, Second Edition, Springer-Verlag, 1983. MR737190 (86c:35035)

[12] J. Hadamard, Mémoire sur le problème d'analyse relatif à l'équilibre des plaques élastiques encastrées, OEuvres de Jacques Hadamard, 515-631, Vol. 2, Ed. CNRS, Paris, 1968.

[13] T. Kato, Perturbation Theory for Linear Operators, Springer-Verlag, New York, 1976. MR0407617 (53:11389)

[14] V. Kozlov, On the Hadamard formula for nonsmooth domains, J. Differ. Equat., 230 (2006), 532-555. MR2269932 (2007h:35244)

[15] Y.Y. Li and M. Vogelius, Gradient estimates for solutions to divergence form elliptic equations with discontinuous coefficients, Arch. Rational Mech. Anal., 153 (2000), 91-151. MR 1770682 (2001m:35083)

[16] Y.Y. Li and L. Nirenberg, Estimates for elliptic systems from composite material, Comm. Pure Appl. Math., 56 (2003), 892-925. MR.1990481(2004k:35097)

[17] J. Osborn, Spectral approximation for compact operators, Math. of Comp., 29 (1975), 712725. MR0383117 (52:3998)

[18] S. Osher and F. Santosa, Level set methods for optimization problems involving geometry and constraints I. Frequencies of a two-density inhomogeneous drum, J. Comput. Phys., 171 (2001), 272-288. MR.1843648 (2002f:65088)

[19] O.S. Salawu, Detection of structural damage through changes in frequency: a review, Engineering Structures, 19 (1997), 718-723. 
[20] J. Sanchez Hubert and E. Sanchez Palencia, Vibration and Coupling of Continuous Systems, Asymptotic Methods, Springer-Verlag, Berlin, 1989. MR996423 (91c:00018)

[21] B. Simon, A canonical decomposition for quadratic forms with applications to monotone convergence theorems, J. Funct. Anal., 28 (1978), 377-385. MR0500266 (58:17937)

[22] P. Stollmann, A convergence theorem for Dirichlet forms with applications to boundary value problems with varying domains, Math. Z., 219 (1995), 275-287. MR1337221 (96d:60119)

[23] J. Weidmann, Continuous dependence of eigenvalues and eigenfunctions of elliptic differential operators on the domain (in German), Math. Scand., 54 (1984), 51-69. MR753063 (86f:35133)

Centre de Mathématiques Appliquées, CNRS UMr 7641 and Ecole Polytechnique, 91128 Palaiseau Cedex, France

E-mail address: ammari@cmapx.polytechnique.fr

Dipartimento di Matematica "G. Castelnuovo" Università di Roma "La Sapienza", Piazzale Aldo Moro 5, 00185 Roma, Italy

E-mail address: beretta@mat.uniroma1.it

Dipartimento di Matematica, Università degli Studi di Firenze "Ulisse Dini", Viale Morgagni 67/A, 50134 Firenze, Italy

E-mail address: francini@math.unifi.it

Department of Mathematics, Inha University, Incheon 402-751, Korea

E-mail address: hbkang@inha.ac.kr

Department of Mathematical Sciences, Korean Advanced Institute of Science and Technology, 335 Gwahangno (373-1 Guseong-dong), Yuseong-gu, Daejeon 305-701, KoREA

E-mail address: mklim@kaist.ac.kr 